\title{
PIPERINE MODULATES NLRP3 / TLR4 / NF-KB SIGNALLING PROCESSES OF ANGIOGENESIS AND NEUROGENESIS IN RATS FOLLOWING ISCHEMIC STROKE
}

\author{
JUN YANG ${ }^{1}$, YANG SHENG ${ }^{1}$, YONG LU² and YUE ZHAO ${ }^{2 *}$
}

${ }^{1}$ Department of Rehabilitation, Hankou Hospital, Wuhan, Hubei, China, 430012

${ }^{2}$ The Third Department of Anus \& Intestine Surgery, The Eighth Hospital of Wuhan, Wuhan, Hubei, China, 430010

\begin{abstract}
Early studies have shown neurotrophic and neuroprotective functions and have grown considerably in the treatment of brain aging-related disorders correlated with age, but their fundamental role against ischaemic stroke remains unclear in MCAO (middle cerebral artery occlusion) induced rats. In this research, we evaluated the influence of piperine on angiogenesis and neurogenesis in ischemic rats and subsequently identified the fundamental mechanism of NF-kB /NLRP3/ TLR4 signaling pathways. In rats, MCAO was conducted to cause ischemic stroke and 2,3,5-Triphenyltetrazolium chloride staining (TTC) staining was utilized to assess if the MCAO procedure was effectively developed. The assessment of neurological disorder was carried out. Immunofluorescence staining was studied to evaluate the influence of neurogenesis and angiogenesis after oral dosing ( 5 or $10 \mathrm{mg} / \mathrm{kg}$ ) of piperine in MCAO triggered rats. Besides, by western blotting analysis, the proteins (Caspase-1, IL-1 $\beta$, NLRP3, TLR4, NF- $\kappa$ B p65) active in the signaling cascade TLR4/NF-2B/NLRP3 were identified. The number of neurons in the infant and brain microvessel density (MVD) following ischemic stroke was greatly improved during the treatment with piperine. Treatment of piperine significantly suppressed Caspase-1, IL-1 $\beta$, NLRP3, TLR4, NF-кB p65 proteins implicated in the signaling process of NF-kB /NLRP3/ TLR4. Piperine improves neurogenesis and angiogenesis by modulation of the inflammatory signaling cascade of NF-kB /NLRP3/ TLR4 after ischemic stroke in rats. Therefore, piperine could be a successful drug for the inhibition of ischemic stroke.
\end{abstract}

Keywords: Piperine, ischemic stroke, microvessel density, angiogenesis, TLR, neurogenesis

Stroke is the world's $5^{\text {th }}$ major cause of death with two subtypes, ischaemic and hemorrhagic stroke $(1,2)$. Eighty-seven percent of the strokes are ischemic strokes with severe pathological occurrences (3). Tissue plasminogen factor (Alteplase) is a major FDA-approved ischaemic stroke treatment with minimal therapeutic window side effects (less than $5 \mathrm{~h}$ ) for thrombolysis (4). Further research on ischemic stroke therapeutic approaches is therefore required. Two main injuries after ischemic stroke include infarction of the heart and penumbra. The penumbra region, apart from the infarct, may to some degree be recovered, if reperfusion is achieved. The emphasis on penumbra intervention will help reduce the infarction zone and dramatically reduce neurological impairment (5).

Neural pharmacological remodeling is an encouraging therapeutic approach for long-term neurological restoration after stroke (6). Improved ischemic stroke-induced neurogenesis and angiogenesis are important for the regulation of neuronal pathways and brain function, which is highly involved in the neurological recovery of long-term functions $(7,8)$.

Neurogenesis ensuing ischemic stroke means that neural precursors cells (NPC) are proliferated, differentiated, and migrated to the affected region for self-regeneration (9). In most cases, distinct cell subtypes, such as premature neurons, astrocytes, oligodendrocytes, and so on, are essential to recovering functions in a wounded brain (10). Angiogenesis is also of significance for the recovery of brain activity after ischemic stroke since this occurrence enhances vascular density and blood flow to the cerebrum (11). Angiogenesis in infected areas is influenced by the proliferation, migration, cell adhesion, and differentiation of endothelial cells (10). Angiogenesis and neurogenesis jointly reinforce neuronal activity following ischemic stroke $(12,13)$. On the one side, newly formed blood vessels have a nourishing

* Corresponding author: e-mail: yuezhao738@gmail.com 
blood supply with a favorable neurogenesis microenvironment. On the other side, the development of the blood vessels is benefitted by newly formed endothelial cells $(14,15)$.

Emergent evidence has demonstrated that neuroinflammation is modulated by the neurogenesis process, including the multiplying, differentiation, and invasion of precursor neural cells (16); Probably, IL-1 $\beta$ has a reasonable effect on neurogenesis inhibition (17). In certain cases, IL-1 $\beta$ release implies that some inflammasomes known as protein complexes are modulated (18). In neuroinflammatory pathogenesis, the nod-like receptor protein 3 (NLRP3) is of considerable importance (19). NLRP3 inflammasome priming requires Nuclear Factor $-\kappa \mathrm{B}(\mathrm{NF}-\kappa \mathrm{B})$ and Toll-like receptor activation (TLRs). Besides, the active NLRP3 assembles nucleated inflammatory compounds, which contribute to caspase- 1 and IL-1 $\beta$ activation to speed up inflammatory mediated cell death through pyroptosis. In specific, NLRP3 reacts to DAMPs (danger-associated molecular patterns), indicating a potential goal for therapeutic strategies (20).

Recently, naturally occurring supplements have gained growing interest as a substitute and complementary treatment for the prevention of neurodegeneration and dementia (21-24). Several natural dietary products, such as pterostilbene, anthocyanin, resveratrol, and curcumin vitamins have been indicated to enhance learning and memory in elderly animals (21, 25-28). Black peppers and other similar herbs are comprised of natural components of piperine. There are numerous biological functions of piperine including immune regulation, cancer prevention, anti-oxidative therapy, anti-inflammatory disorder, and anti-asthma (29). Piperine has been systematically investigated as a supporting ingredient for other substances such as CoQ10, curcumin, and resveratrol. Besides, oxidative stress and inflammation prevention by piperine has been documented (30-32). It has often been noted in the treatment of aging brains and central nervous system diseases of age-associated neurotrophic and neuroprotective behaviors (31, 33-36). Piperine suppressed ERK or JNK signaling and hindered inflammatory reaction to dendritic cells for immune regulation (37). Piperine may also target type I interferon production and antagonize endotoxin shock triggered by LPS (38). Liang et al. established that piperine greatly inhibited IL-1 $\beta$ and HMGB1 (high-mobility box-1) protein production and pyroptosis in LPS-mediated macrophages by suppressing the stimulation of AMPK (ATP-induced protein kinase) (39). Furthermore, piperine decreased IL1 $\beta$, TNF- $\alpha$, and NLRP3 levels effectively, which improve diabetic nephropathy (40). We investigated the impact of piperine on neurogenesis after ischaemic stroke in rats in our research. In addition, the underlying mechanism of the signaling pathway TLR/NF-kB/NLRP3 was investigated.

\section{EXPERIMENTAL}

\section{Animals}

Male, adult, and healthy rats (280-300 g) were procured from the Research Center, China. All rats were kept at room temperature with $12 \mathrm{~h}$ lights/12 h dark, in accordance with the national guidelines for the maintenance and care of the laboratory animals. Filtered drinking water and normal animal diets were fed to the animals. Prior approval of the Animal Ethics Committee was obtained for all experimental procedures. All surgeries were carried out under aseptic conditions.

\section{Surgical procedure involving middle cerebral artery occlusion}

The MCAO activity was performed according to the method described by Zea Longa (41). In short, Sprague-Dawley rats were anesthetized using acepromazine maleate $(5 \mathrm{mg} / \mathrm{kg})$ and ketamine hydrochloride $(80-100 \mathrm{mg} / \mathrm{kg}$ ) and the common right, external and internal carotid arteries (CCA, ECA \& ICA) were carefully excised via the middle incision of the neck. Before a minimal resistance to obscure blood flow of the middle carotid artery was detected, care was taken with a monofilament nylon suture of 4.0 (0.24 $\mathrm{mm}$ in diameter) with a circular silicone edge of the CCAs in the ICA. The nylon suture was then tied to ensure that it did not retreat spontaneously. Nylon suture was removed after one hour of occlusion for the reperfusion of blood. Without the MCAO, the sham group had identical operating procedures

\section{Drug treatment}

The rats were allocated into four categories: Sham group, MCAO group ( $0.5 \%$ sodium carboxymethylcellulose), and MCAO + Piperine groups ( $5 \mathrm{mg} / \mathrm{kg}$ and $10 \mathrm{mg} / \mathrm{kg}$ respectively). Intragastrically piperine (5 and $10 \mathrm{mg} / \mathrm{kg}$, obtained from Sigma Aldrich, Beijing China; CAS No. 94-62-2) was administered for fourteen successive days after ischemic stroke induction. Intragastrically, $0.5 \%$ sodium carboxymethylcellulose was administered for 14 days to the sham and MCAO groups. After seven days of ischemic stroke inductions, the thymidine analog (BrdU, 5-Bromo-2-deoxyuridine, $50 \mathrm{mg}$ per $\mathrm{kg}$ ) was injected intraperitoneally once daily for seven successive days to obtain newly formed cells. 


\section{TTC (2, 3, 5-Triphenyltetrazolium chloride) staining}

A TTC staining was conducted to determine if middle cerebral artery occlusion procedures were effective upon evaluating infarction areas between the sham and the vehicle-treated groups as previously stated (42). In short, cardiac perfusion was performed $24 \mathrm{~h}$ after reperfusion of blood, under deep anesthesia, followed by easy removal and freezing of the rat's brains at $-20^{\circ} \mathrm{C}$. The brains were sliced into 5 sections after $30 \mathrm{~min}$ and then incubated for $40 \mathrm{~min}$ at room temperature in 2 percent of TTC. The five portions were dipped in 5\% paraformaldehyde for one hour and photographed.

\section{Evaluation of neurological deficit}

The assessment of neurological deficit was conducted following reperfusion on the $1^{\text {st }}, 5^{\text {th }}, 10^{\text {th }}$, and $14^{\text {th }}$ day using the Zea Longa method (41). The following criteria were used: 0 , average, no neurologic impairment; 1, mild neurological disability, inability to entirely stretch the left forepaw; 2 , moderate; neurological disability, left rounding; 3 , severe neurological disability falling from left; 4 , depressed-conscious with repetitive involuntary gestures; 5, death. MCAO-operated rats scored between 1 and 3 .

\section{Immunofluorescence staining}

After 14 days of treatments, the rats were sufficiently anesthetized and the brains were extracted from the chest with ice-cold phosphate buffer saline. The brains were then instantly implanted into the Tissue-Tek OCT and sliced into $5 \mu \mathrm{m}$ thick sections. The BrDU (5'-Bromo-2'-deoxyuridine) was profoundly stained and the neuronal nuclei (neuron marker, NeuN) were conducted to identify penumbra neurogenesis after MCAO. In short, the frozen slices were denatured at room temperature for $40 \mathrm{~min}$ with $2 \mathrm{M}$ hydrochloric acid and blocked at room temperature for one hour with $4.5 \%$ normal goat serum in phosphate buffer saline. Then the slides were incubated for $4^{\circ} \mathrm{C}$ overnight against NeuN (1 : 3000, Abcam, UK) and BrdU's (1 : 300, Cell Signaling Technology, USA) primary antibodies. Later, Alexa Fluor 594 and 488 (1: 500, Abcam, UK) were used to incubate the slides. InForm ${ }^{\circledR}$ software was used to digitize the images.

To visualize the vascular density, the immunofluorescence staining method was used to identify angiogenesis throughout the penumbra following MCAO. The brain vessel was labeled with the CD31. In brief, 5 percent normal serum of goat in phosphate buffer saline containing $0.5 \%$
Tween 80 for $1 \mathrm{~h}$ was used to block the sliced sections and incubated overnight at $4^{\circ} \mathrm{C}$ against antiCD31 mouse (1 : 50; Abcam, UK). Later, Alexa Fluor 594 ( 1 : 500, Abcam, UK) was used to incubate the slides. Besides, all sliced sections were counterstained with DAPI (4, 6-diamidino-2-phenylindole). The images were digitized with the InForm $^{\circledR}$ software with a multi-spectral imaging system (PerkinElmer, USA).

\section{Western blot analysis}

The western blotting analysis was carried out as previously stated by the protocol (43). The RIPA lysis buffer was used to extract the brain tissue protein from ischemic penumbra and the BCA kit was used to detect protein concentration. The primary antibodies (Cell Signaling Technology, Shanghai, China) were incubated for overnight as followsAnti- $\beta$-tubulin (1 : 500); Anti-NF- $\kappa \mathrm{B}$ p65 and Anti-p-NF- $\kappa$ B p65 (1 : 1000); Anti-TLR4 antibody (1 : 300); Anti-NLRP3 antibody (1: 1000); AntiIL-1 $\beta$ antibody (1:1000); Anti-pro-Caspase- 1 and Anti-Caspase-1 (1:1000 and 1:2000 respectively), Anti- $\beta$-actin antibody $(1: 4000)$. Visualization of the protein bands was done using the enhanced chemiluminescence (ECL) method after proper incubation against secondary antibodies and computed by Image Pro-Plus software.

\section{Statistical analysis}

For statistical analysis, GraphPad Prism 7 was used. The data were reported as mean $\pm \mathrm{SD}$, followed by the differences evaluated by ANOVA one-way and by t-test. A significant difference was found to be $\mathrm{p}<0.05$.

\section{RESULTS}

\section{Neurological deficit evaluation followed by TTC staining}

A TTC staining for sham and vehicle-treated (model) groups' rats was seen in Figure 1A. The vehicle-treated rats displayed a large infarct size showing the grey area relative to the sham group which shows that the model MCAO was successful. Figure 1B showed neurological deficit evaluation. To a certain extent, MCAO surgery conducted in rats without drug administration was able to recover on themselves slowly, but in the model group, there was no substantial difference. Neurological dysfunction was reduced subsequently on 5, 10, and 14 days of piperine intervention ( 5 and $10 \mathrm{mg} / \mathrm{kg}$ respectively). Of these days, neurological dysfunction evaluation had dramatically decreased following ten $(\mathrm{p}<0.05)$ 
and fourteen $(\mathrm{p}<0.01)$ days of piperine treatment respectively $(10 \mathrm{mg} / \mathrm{kg})$.

\section{Influence of piperine on neurogenesis after ischemic stroke in the penumbra}

To decide if endogenous neurogenesis might enhance piperine intervention after ischemic stroke over 14 days, we utilized NeuN (the indicator for mature neurons) to corroborate endogenous neurogenesis. The BrdU was also administered in the peritoneal cavity to mark the newly formed cells. The number of positive cells $\mathrm{NeuN}+/ \mathrm{BrdU}+$ in the vehicle group was significantly $(\mathrm{P}<0.01)$ higher when compared to the sham group. Nevertheless, the number of positive cells ( $\mathrm{NeuN}+/ \mathrm{BrdU}+$ ) in piperine treated ( 5 and $10 \mathrm{mg} / \mathrm{kg}$ ) groups were substantially enhanced relative to the vehicle group. Furthermore, the newly multiplying neurons (labeled as $\mathrm{NeuN}$ ) were quantified in the penumbra, counting the total of positive cells $(\mathrm{NeuN}+/ \mathrm{BrdU}+)$. The piperine-treated groups
A)

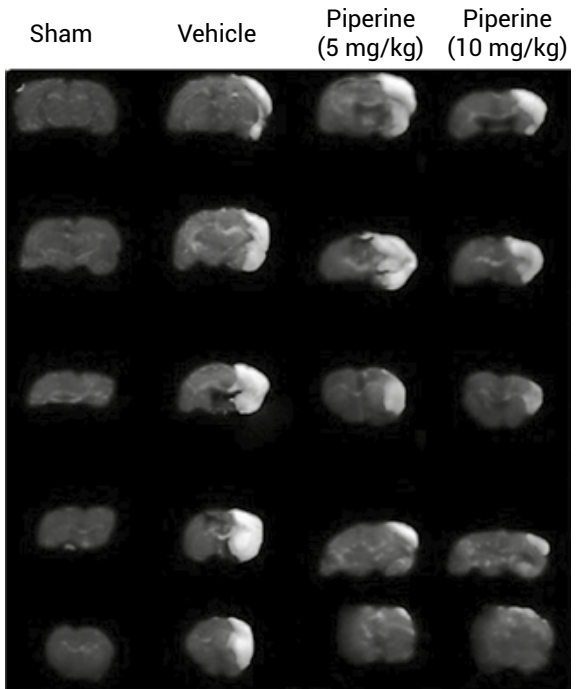

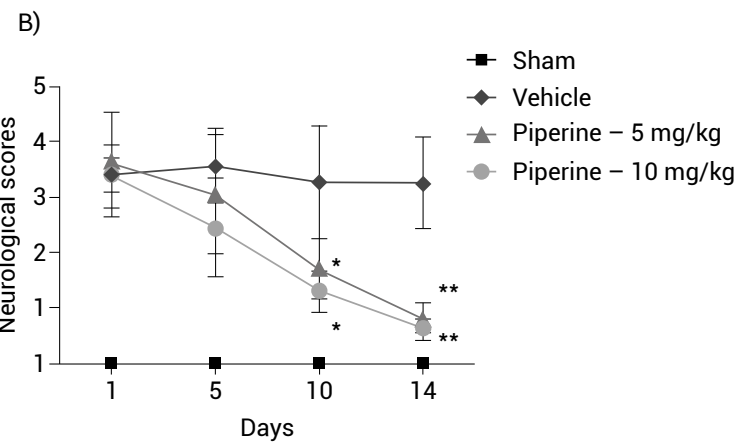

Figure 1. Neurological deficit assessment after cerebral ischemic injury for 14 days in rats. A) Representative TTC staining images in the sham group and vehicle-treated group after cerebral ischemic injury. White area: infarct area; Red area: normal area. B) Neurological deficit evaluation was conducted after $1,5,10,14$ days of middle cerebral artery occlusion. ${ }^{*} \mathrm{p}<0.05$ and $* * \mathrm{p}<0.01$, compared with the vehicle groups respectively.
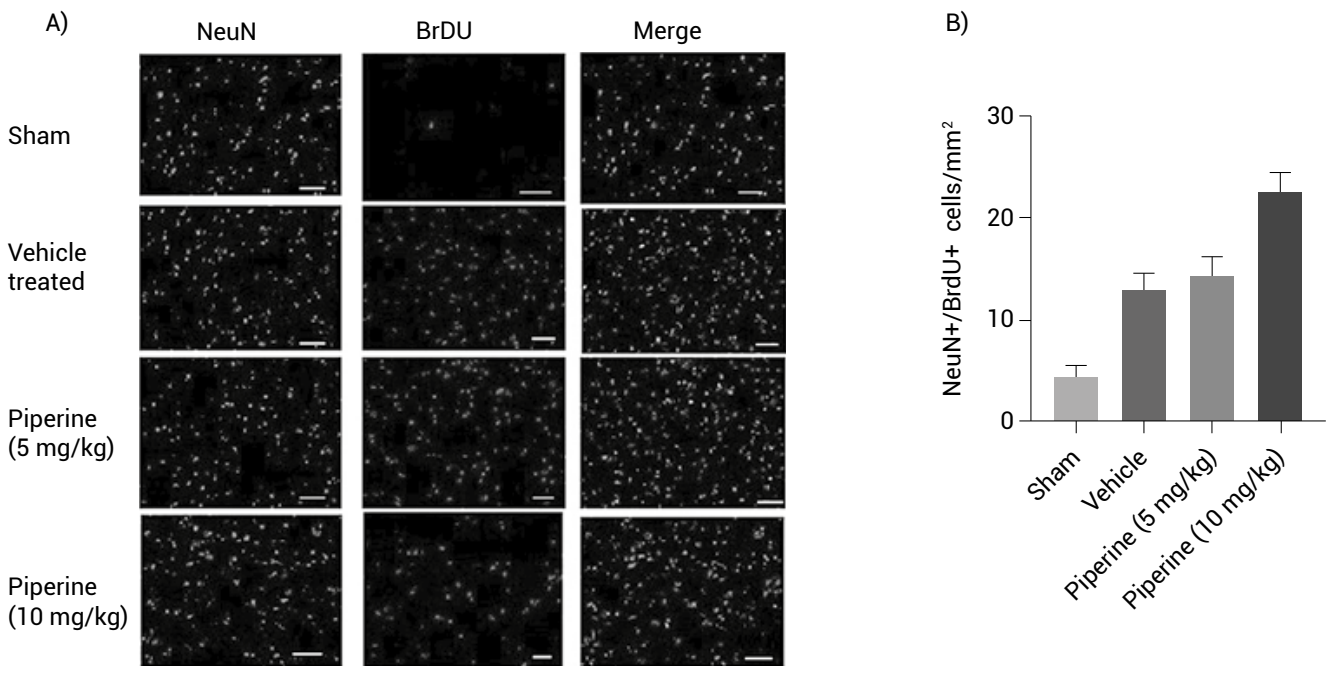

Figure 2. Piperine enhances neurogenesis in the penumbra of rats after cerebral ischemic injury for 14 days. Rats underwent MCAO surgery and were treated with piperine $(5$ or $10 \mathrm{mg} / \mathrm{kg}$ ) for 14 days. The brain tissue was cut into $5 \mu \mathrm{m}$ and the section from the penumbra of the rats was digitalized. NeuN (a marker for mature neurons) was used as a marker to demonstrate endogenous neurogenesis. BrDu was intraperitoneally injected to label the newborn cells. A) Representative immunofluorescence image showing co-localization of NeuN - stained cells (green) and BrdU-labeled cells (red). NeuN, Neuronal Nuclei; BrdU, 5'-bromo-2'-deoxyuridine. B) Quantification of NeuN*/BrdU co-labeling cells in different groups, $\mathrm{n}=8$ per group. $\# \mathrm{p}<0.01$, compared to the sham group, ${ }^{* *} \mathrm{p}<0.01, * * * \mathrm{p}<0.001$, compared with the vehicle group. Scale bar, $100 \mu \mathrm{m}$. 
had significantly augmented the $\mathrm{NeuN}+/ \mathrm{BrdU}+$ cells in the penumbra, especially in the piperine group treated with $10 \mathrm{mg} / \mathrm{kg}$ as compared with the vehicle group $(\mathrm{p}<0.01)$. These findings indicated that piperine could promote the neuronal progenitor's proliferation in the penumbra following an ischemic stroke after 14 days and assisted the invasion of newly formed neuronal cells into the location of lesions. Figure 2 showed the results of $\mathrm{NeuN}+/ \mathrm{BrdU}+$ double stained in the penumbra.

\section{Influence of piperine on angiogenesis after ischemic stroke in the penumbra}

For cerebral flow and neurogenesis, vascular density is vital. Brain vascular densities were decreased substantially $(\mathrm{p}<0.01)$ in MCAO-induced rats relative to the sham group. However, vascular density, in the piperine-treated group at $10 \mathrm{mg} / \mathrm{kg}$ $(p<0.05)$ was increased. Figure 3 shows the effects of vascular density.

\section{Influence of piperine on NF-кB and TLR4-signalling pathway activation}

The animals were sacrificed and the penumbra's tissues were collected for Western blotting analysis to examine the expression of NF- $\kappa \mathrm{B}$ and TLR-4 after the MCAO procedure. The TLR4 content in the vehicle-treated group was considerably increased $(p<0.05)$ relative to the sham group. However, TLR4 expression was reduced in MCAO
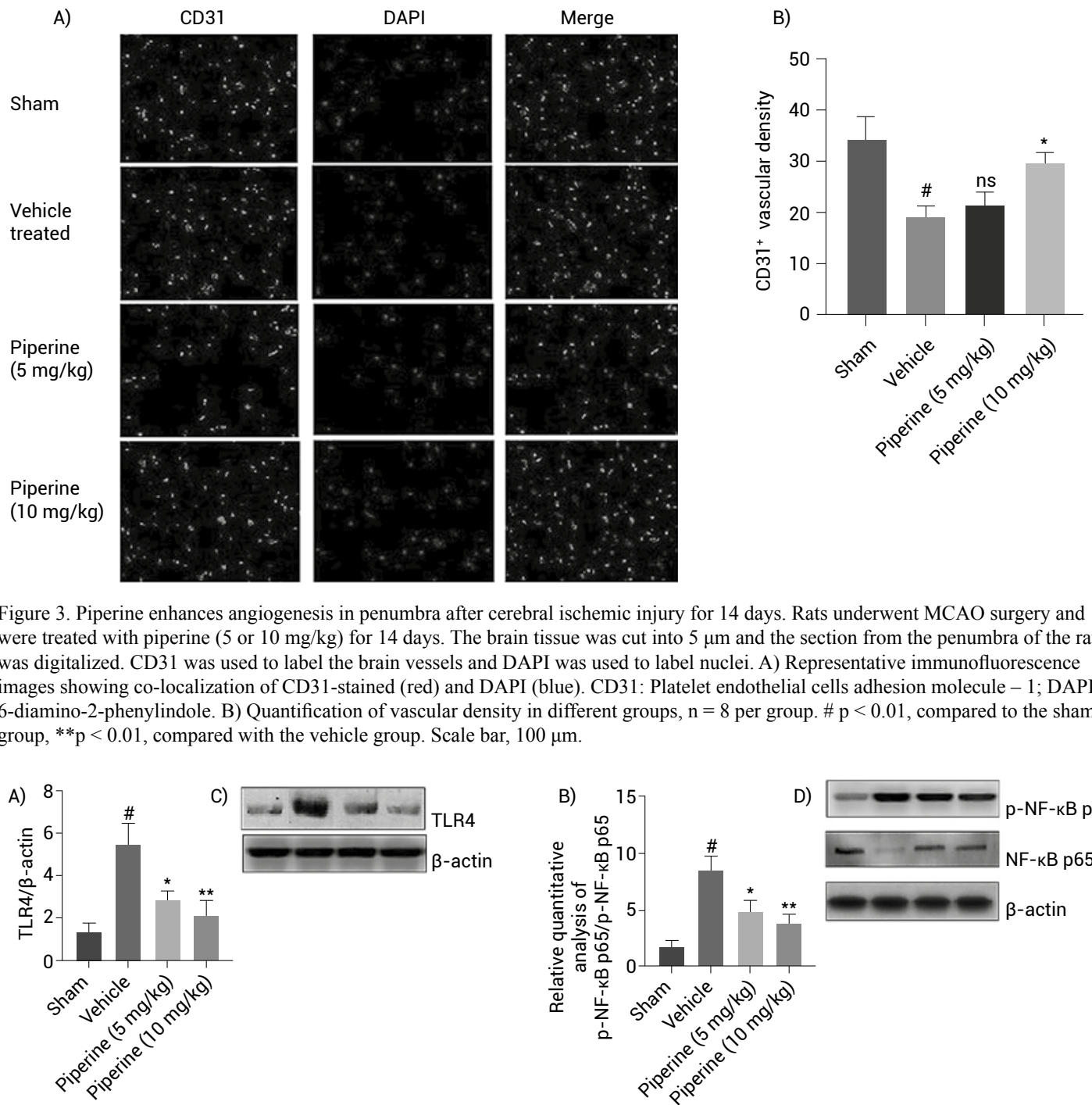

Figure 4. Effect of piperine on inhibition of TLR4 and NF- $\kappa B$ signaling pathway. Rats underwent MCAO surgery and were treated with piperine $(5-10 \mathrm{mg} / \mathrm{kg}$ ) for 14 days. The rat brain tissue from the penumbra was extracted by RIPA. (A) \& (B) Quantification of TLR4, nuclear factor $-\kappa B(N F-\kappa B)$ and phosphorylation of nuclear factor- $\kappa B$ expression. $\beta$-actin was used as a loading control. (C) \& (D) Representative western immunoblotting analysis of TLR4, nuclear factor $-\kappa \mathrm{B}(\mathrm{NF}-\mathrm{\kappa B})$ and phosphorylation of nuclear factor- $\mathrm{\kappa B}$ expression $(\mathrm{p}-\mathrm{NF}-\mathrm{\kappa B}), \mathrm{n}=8$. $\# \mathrm{p}<0.01$, compared to the sham group, ${ }^{*} \mathrm{p}<0.05,{ }^{*} \mathrm{p}<0.01$, compared with the vehicle group. 
rats following piperine treatment. Significant variance $(\mathrm{p}<0.05)$ was noticed in the piperine treated group at $10 \mathrm{mg} / \mathrm{kg}$. Piperine treatment was shown to prevent the TLR-4 signaling pathway activation. In comparison with the sham group, the NF- $\mathrm{B}$ p65/ $\mathrm{p}-\mathrm{NF}-\kappa \mathrm{B}$ p65expression in the vehicle-treated group was significantly enhanced, suggesting the activation of the signaling pathway of the NF- $\kappa$ B following the MCAO procedure. Nonetheless, substantial lesser $(\mathrm{p}<0.01)$ expression in piperine treated groups (5 and $10 \mathrm{mg} / \mathrm{kg}$ ) of p-NF- $\kappa \mathrm{B}$ p $65 / \mathrm{NF}-\kappa \mathrm{B}$ p 65 was reported which suggested inhibition of the NF- $\kappa B$ pathway after piperine treatment. The findings were presented in Figure 4.

\section{Influence of piperine on NLRP3-signaling pathway activation}

Because of the underlying mechanisms that the development of IL-1ß involves the stimulation of inflammasome of the NLRP3 and Caspase-1, expression from western blotting of NLRP3, Caspase-1, pro-Caspase-1, IL-1 $\beta$, and pro-IL-1 $\beta$ tissue was examined. Figure 5 shows a surprisingly higher expression $(p<0.01)$ of IL-1 $\beta$ after rats were exposed to MCAO activity. The level of IL-1 $\beta$ in piperine treatment $(10 \mathrm{mg} / \mathrm{kg})$ compared to the vehicle group, however, was significantly lower $(\mathrm{p}<0.05)$ which indicates that piperine may have a potential role in inhibiting the development of IL1 $\beta$. Meanwhile, when the MCAO-operated rats were exposed to pro-IL-1 $\beta$, an IL-1 $\beta$ precursor, significantly $(\mathrm{p}<0.01)$ enhanced. Pro-IL-1 $\beta$ levels also reduced considerably in the piperine-treated group at a dose of $10 \mathrm{mg} / \mathrm{kg}$. Besides, the NLRP3 expression in the vehicle-treated $(\mathrm{p}<0.01)$ group relative to sham was substantially increased. Nevertheless, after piperine treatment, NLRP3 was considerably reduced, particularly in the piperine treatment at $10 \mathrm{mg} / \mathrm{kg}$ group $(\mathrm{p}<0.01)$, signifying that piperine could play a potential role in inhibiting the development of NLRP3. Furthermore, the pro-Caspase-1 expression in the vehicle-treated group was remarkably increased and decreased in the piperine-treated groups considerably (5 or $10 \mathrm{mg} / \mathrm{kg}$ ). The findings showed that piperine treatment substantially inhibited the inflammatory signaling pathway TLR4/NF- $\kappa \mathrm{B} / \mathrm{NLRP}$ in MCAO rats.

\section{DISCUSSION}

A blocked cerebral artery contributes to ischemic stroke, which is a significant reason for death causing an enormous social burden worldwide (10). Increasingly therapeutic approaches established for ischemic stroke were unsuccessful in clinical studies owing to the intricacy of the CNS (central nervous system) (43). Also, ischemic stroke-inducing neurological deficits is a lasting process. Safe and effective agents need to be developed to treat ischemic stroke (44). The neuroprotective mechanism of piperine, however, remains uncertain. Adult neurogenesis progresses in the damaged brain regions of SVZ (subventricular) and dentate gyrus (DG) but seldom in the physiological state (9). Similarly, neurogenesis following CNS disease without any intervention is
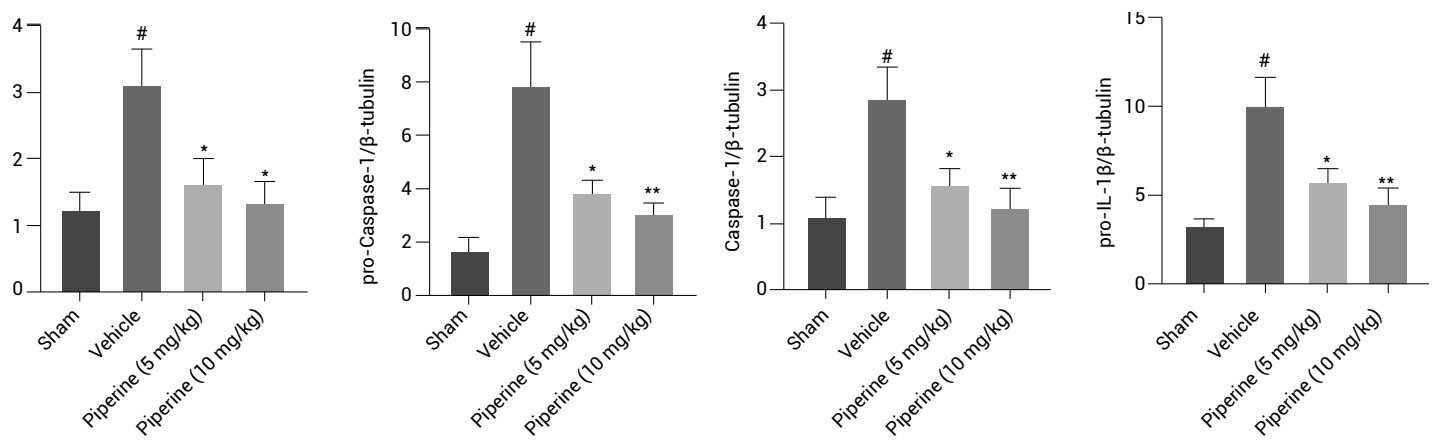

Figure 5. Effect of piperine on inhibition of NLRP3 signaling pathway. Rats underwent MCAO surgery and were treated with piperine ( 5 or $10 \mathrm{mg} / \mathrm{kg}$ ) for 14 days. The rat brain tissue from the penumbra was extracted by RIPA. A) Quantification of NLRP3, pro-caspase -1, caspase-1, pro-Il-1 $\beta$, IL-1 $\beta$. $\beta$-tubulin was used as a loading control. B) Representing Western blot analysis of NLRP3, pro-caspase -1 , caspase-1, pro-Il-1 $\beta$, IL-1 $\beta$. \# $\mathrm{p}<0.01$, compared to the sham group, $* \mathrm{p}<0.05$,
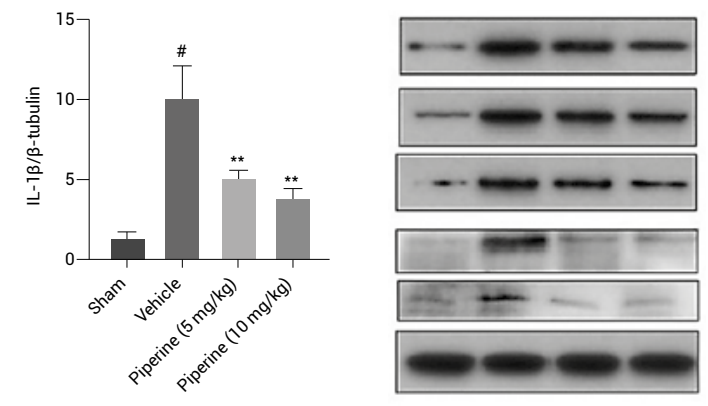

NLRP3 $* * \mathrm{p}<0.01$, compared with the vehicle group 
too difficult to restore brain insults (45). Therefore, pharmacological agents will be useful for facilitating this mechanism to persistently promote neurogenesis (46). Angiogenesis mediated by the therapeutic compounds is also important for accelerating cerebral blood flow and producing a favorable neurogenesis situation. Also, increased neurogenesis is accompanied by a higher density of microvessels (MVD) (47). In our findings, we effectively identified and improved steady middle brain artery occlusion (MCAO) surgical procedures (Figure 2A) for further research to investigate the neurogenesis and angiogenesis actions of the piperine following ischemic stroke during two weeks of neurological deficit evaluation.

The neurological deficit decreased significantly following oral administration of piperine, suggesting piperine's positive therapeutic implications on ischemic stroke. The sham group had almost no newborn neurons, indicating that neurogenesis seldom happens under physiological conditions. Neuron number, nevertheless, necrotize and die consistently in the penumbra region, while neurogenesis was induced in the penumbra area after ischemic stroke. Surprisingly, following the piperine effect, the number of newly formed neurons was substantially improved, indicating that piperine is very important in combatting brain damage by encouraging endogenous neurogenesis (Figure 3). A reduced amount of middle vessel density was also found after cerebral ischemic damage in the penumbra region. Farther intriguing, piperine could increase middle vessel density in the penumbra region through angiogenesis following cerebral ischemic damage, additional nutrient blood flow, and the favorable neurogenesis microenvironment (Figure 4). Emerging studies have shown that the neurogenesis mechanism is affected by IL-1 $\beta$ (48-50).

Besides, inflammatory response to neurological deficits is closely linked to releases of certain endogenous ligands that act through toll-like receptors (TLRs). Neuroinflammation mediated by TLR4 is central to

Figure 6. Experimental scheme of Piperine modulating NLRP3/ TLR4/NF- $\kappa \mathrm{B}$ signaling processes of angiogenesis and neurogenesis in rats following ischemic stroke. the induction of secondary brain damage $(51,52)$. Then, through the signal transduction of TLR4 (53) the NF- $\kappa$ B pathway and the NLRP3 inflammasome were triggered. Subsequently, to increase brain insults, IL-1 $\beta$ (a pro-inflammatory cytokine) downstream involved in the NF- $\kappa \mathrm{B}$ pathway, is augmented. Pattern recognition receptors (PRRs) which stimulate NF- $\kappa \mathrm{B}$ and TLRs, are used to primate NLRP3. Inflammasome assembling NLRP3 could induce IL-1 $\beta$ and Caspase-1 functions (54).

While it is evident that both acute and chronic inflammatory states have demonstrated the role of NLRP3, however, it remains unidentified in its underlying mechanism (55). We demonstrated whether piperine improved angiogenesis and neurogenesis through the inhibition of the TLR4/NF- $\mathrm{KB} / \mathrm{NLRP} 3$ signaling pathway. Based on this principle, expression of related proteins includes a substantial increase in signaling pathways such as Caspase-1, IL-1 $\beta$, NLRP3, TLR4, NF-кB p65, pro-Caspase-1, $\mathrm{p}-\mathrm{NF}-\kappa \mathrm{B}$ p 65 , pro- IL-1 $\beta$ following middle carotid artery occlusion surgery without drug treatment for two weeks, suggesting that the signaling pathway TLR4/NF- $\kappa \mathrm{B} / \mathrm{NLRP} 3$ was triggered after an ischemic stroke (Figure 5, Figure 6). Interestingly, piperine may reduce these protein expressions and offer an effective environmental function for neurogenesis and angiogenesis. Piperine is, therefore, a favorable
Male rats were procured and maintained at room temperature $-12 \mathrm{~h} / 12 \mathrm{~h}$ light and dark. Surgeries conducted under aseptic conditions

\section{$\downarrow$}

MCAO activity was performed in anaesthetized rats using Acepromazine maleate $(5 \mathrm{mg} / \mathrm{kg})$ and Ketamine hydrochloride $(80-100 \mathrm{mg} / \mathrm{kg})$ and induced middle carotid artery occlusion

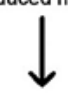

Animals were divided into four groups- sham, Vehicle treated (MCAO group), MCAO+piperine (50 $\mathrm{mg} / \mathrm{kg}$ ) and MCAO (100 mg/kg) group. Intragastric piperine was given for 14 successive days after ischemic stroke induction followed by BrdU, 5-Bromo-2-deoxyuridine was injected intraperitoneally, once daily for 7 successive days

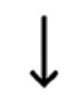

TTC staining was performed followed by neurological deficit evaluation, immunofluorescence staining, and western blot analysis

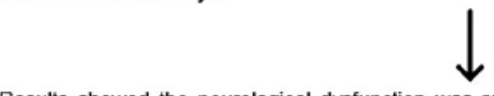

Results showed the neurological dysfunction was reduced subsequently on 5,10 and 14 days of piperine intervention ( 5 and $10 \mathrm{mg} / \mathrm{kg}$ respectively). Vascular density, in the piperine treated group at $10 \mathrm{mg} / \mathrm{kg}(\mathrm{p}<0.05)$ was increased followed by the reduction of TLR4 expression. The pro-Caspase-1 decreased in the piperine treated groups considerably $(5 \mathrm{or} 10 \mathrm{mg} / \mathrm{kg})$.

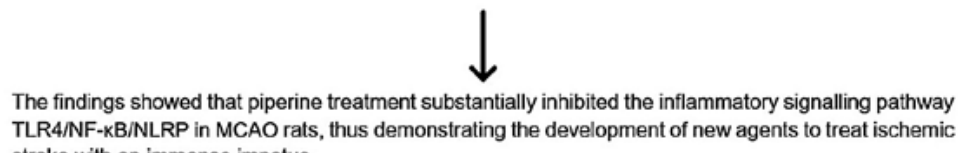
stroke with an immense impetus. 
candidate with the inhibition of the TLR4/NF- $\kappa \mathrm{B} /$ NLRP3 signaling pathways for therapy of ischemic stroke. It is worth noting that the inflammasome of NLRP3, which is an essential immune node in the inborn immune system, is a rapid response to chronic inflammation (56). Therefore it is worthy for clinical application to aim NLRP3 inflammasome for the treatment for ischemic stroke.

\section{CONCLUSIONS}

With these findings of neurogenesis and angiogenesis, piperine could signify advantageous ischemic stroke intervention and treatment. Besides, our results showed that the piperine treatment inhibits the signaling pathway TLR4/NF-אB/NLRP3 to perform its activity. Further studies should be carried out to determine the precise mechanism of activation of inflammasome NLRP3, which is critical for the identification of new targets for effective compounds. The development of new agents to treat ischemic stroke has an immense impetus.

\section{Acknowledgments}

This study was supported by Health Commission of Hubei Province (No. WJ2019F009) and Wuhan Municipal Health Commission (No. WX19D53).

\section{Conflicts of interest}

Authors declare no conflicts of interest in any part of the manuscript submission

\section{REFERENCES}

1. Vaugham A., Yang Q., Tong X.: MMWR Morb. Mortal. Wkly Rep. 66, 933 (2017).

2. Zhou Z., Lu J.: Pharmacol. Ther. 191, 23 (2018).

3. Bennette D.A., Connor M.: Glob. Heart 9, 107 (2014).

4. Vidale S., Agostoni E.: Brain 137, e281 (2014).

5. Anderson C.S., Huang Y., Lindley R.I., Chen X., Arima H., et al.: Lancet 393, 877 (2019).

6. Koh S.H., Park H.H.: Transl. Stroke Res. 8, 3 (2017).

7. Borsini A., Zunszain P.A., Thuret S., Pariante C.M.: Trends Neurosci. 38, 145 (2015).

8. Jin X., Wang R.H., Wang H., Long C.L., Wang H.: Acta Pharmacol. Sin. 36, 1416 (2015).

9. Zhao C., Deng W., Gage F.H.: Cell 132, 645 (2008).

10. Zhang Z.G., Chopp M.: Lancet Neurol. 8, 491 (2009).
11. Seto S.W., Chang D., Jenkins A., Bensoussan A., Kiat H.: J. Clin. Med. 5, 16 pages (2016).

12. Jin K., Zhu Y., Sun Y., Mao X.O., Xie L., et al.: Proc. Natl. Acad. Sci. U. S. A. 99, 11946 (2002).

13. Teng H., Zhang Z.G., Wang L., Zhang R.L., Zhang L., et al.: J. Cereb. Blood Flow Metab. 28, 764 (2008).

14. Licht T., Rothe G., Kreisel T., Wolf B., Benny O., et al.: Proc. Natl. Acad. Sci. U. S. A. 113, E7828 (2016).

15. Palmer T.D., Willhoite A.R., Gage F.H.: J. Comp. Neurol. 425, 479 (2000).

16. Basu A., Lazovic J., Krady J.K., Mauger D.T., Rothstein R.P., et al.: J. Cereb. Blood Flow Metab. 25, 17 (2005).

17. Koprich J.B., Reske-Nielsen C., Mithal P., Isacson O.N.: J. Neuroinflammation. 5, 8 (2008).

18. Franchi L., Muñoz-Planillo R., Núñez G.: Nat. Immunol. 13, 325 (2012).

19. Du R.H., Wu F.F., Lu M., Shu X.D., Ding J.H., et al.: Redox Biol. 9, 178 (2016).

20. Xu C., Lu Z., Luo Y., Liu Y., Cao Z., et al.: Nat. Commun. 9, 4092 (2018).

21. Poddar J., Pradhan M., Ganguly G., Chakrabarti S.: J. Chem. Neuroanat. 95, 70 (2019).

22. Ho Y.S., So K.F., Chang R.C.: Ageing Res. Rev. 9, 354 (2010).

23. Perry E., Howes M.J.: CNS Neurosci. Ther. 17, 683 (2011).

24. Mao J., Huang S., Liu S., Feng X.L., Yu M., et al.: Aging Cell 14, 784 (2015).

25. Joseph J.A., Fisher D.R., Cheng V., Rimando A.M., Shukitt-Hale B.: J. Agric. Food Chem. 56, 10544 (2008).

26. Yu S.Y., Zhang M., Luo J., Zhang L., Shao Y., et al.: Prog. Neuropsychopharmacol. Biol. Psychiatry 45, 47 (2013).

27. Li X.M., Zhou M.T., Wang X.M., Ji M.H., Zhou Z.Q., et al.: J. Mol. Neurosci. 52, 286 (2014).

28. Pacheco S.M., Soares M.S.P., Gutierres J.M., Gerzson M.F.B., Carvalho F.B., et al.: J. Nutr. Biochem. 56, 193 (2018).

29. Meghwal M., Goswami T.K.: Phytother. Res. 27, 1121 (2013).

30. Wang-Sheng C., Jie A., Jian-Jun L., Lan H., Zeng-Bao X., et al.: Int. Immunopharmacol. 42, 44 (2017).

31. Mao Q.Q., Huang Z., Ip S.P., Xian Y.F., Che C.T.: Cell Mol. Neurobiol. 32, 531 (2012).

32. Selvendiran K., Sakthisekaran D.: Biomed. Pharmacother. 58, 264 (2004).

33. Mao Q.Q., Huang Z., Zhong X.M., Xian Y.F., Ip S.P.: Behav. Brain Res. 261, 140 (2014).

34. Mao Q.Q., Huang Z., Zhong X.M., Xian Y.F., Ip S.P.: Neurochem. Int. 74, 36 (2014). 
35. Chonpathompikunlert P., Wattanathorn J., Muchimapura S.: Food Chem. Toxicol. 48, 798 (2010).

36. Yang W., Chen Y.H., Liu H., Qu H.D.: Int. J. Mol. Med. 36, 1369 (2015).

37. Bae G.S., Kim J.J., Park K.C., Koo B.S., Jo I.J., et al.: Phytother. Res. 26, 1893 (2012).

38. Bae G.S., Kim M.S., Jung W.S., Seo S.W., Yun S.W., et al.: Eur. J. Pharmacol. 642, 154 (2010).

39. Liang Y.D., Bai W.J., Li C.G., Xu L.H., Wei H.X., et al.: Front Pharmacol. 7, 390 (2016).

40. Samra Y.A., Said H.S., Elsherbiny N.M., Liou G.I., El-Shishtawy M.M., et al.: Life Sci. 157, 187 (2016).

41. Longa E.Z., Weinstein P.R., Carlson S., Cummins R.: Stroke 20, 84 (1989).

42. Lin T.N., He Y.Y., Wu G., Khan M., Hsu C.Y.: Stroke 24, 117 (1993).

43. Fann D.Y., Lee S.Y., Manzanero S., Chunduri P., Sobey C.G., et al.: Ageing Res. Rev. 12, 941 (2013).

44. Jiang T., Wu M., Zhang Z., Yan C., Ma Z., et al.: Mol. Med. 25, 22 (2019).

45. Ernst A., Alkass K., Bernard S., Salehpour M., Perl S., et al.: Cell 156, 1072 (2014).
46. Ruan L., Wang B., Zhuge Q., Jin K.: Brain Res. 1623, 166 (2015).

47. Arai K., Jin G., Navaratna D., Lo E.H.: FEBS. J. 276, 4644 (2009).

48. Dowlati Y., Herrmann N., Swardfager W., Liu H., Sham L., et al.: Biol. Psychiatry 67, 446 (2010).

49. Krieglstein K., Zheng F., Unsicker K., Alzheimer C.: Trends Neurosci. 34, 421 (2011).

50. Pisanu A., Lecca D., Mulas G., Wardas J., Simbula G., et al.: Neurobiol. Dis. 71, 280 (2014).

51. Ma M., Pei Y., Wang X., Feng J., Zhang Y., et al.: Cell Prolif. 52, e12525 (2019).

52. Rodríguez-Yáñez M., Brea D., Arias S., Blanco M., Pumar J.M., et al.: J. Neuroimmunol. 247, 75 (2012).

53. Bauernfeind F.G., Horvath G., Stutz A., Alnemri E.S., MacDonald K., et al.: J. Immunol. 183, 787 (2009).

54. Hanamsagar R., Torres V., Kielian T.: J. Neurochem. 119, 736 (2011).

55. Mangan M.S.J., Olhava E.J., Roush W.R., Seidel H.M., Glick G.D., et al.: Nat. Rev. Drug Discov. 17, 588 (2018).

56. Próchnicki T., Latz E.: Cell Metab. 26, 71 (2017). 